\title{
Practical Capacity Calculation for Time-Hopping Ultra-wide Band Multiple-Access Communications
}

\author{
Abdulkareem Adinoyi and Halim Yanikomeroglu
}

\begin{abstract}
In this letter the practical capacity, known as the cutoff rate, of time-hopping (TH) ultra-wide band (UWB) communication system is evaluated for multiple-access channels. The cutoff rate can be used for determining various system tradeoffs. For instance, it is shown in this letter that if synchronization problems would preclude high spreading factors, a suitable number of hops can be used instead to achieve the same performance. Moreover, it is demonstrated that the cutoff rate evaluated here can be a fast way of gaining insights into the multiuser capacity of TH-PPM UWB systems.
\end{abstract}

Index Terms-Cutoff rate, ultra-wide band (UWB), timehopping, multiple-access channel.

\section{INTRODUCTION}

$\mathbf{C}$ ONSIDERABLE interest has developed for timehopping (TH) ultra-wide band (UWB) multiple-access communication systems [1]. This is due to UWB's appealing features; for instance, it does not require a sinusoidal carrier, it can highly resolve multipath, and it enjoys low probability of detection and interception. These features make UWB technology a promising option for high data rate communications.

The error exponent and cutoff rate are practical and important information-theoretic measures used extensively in the literature for comparing coding scheme performance [2] and constellation design [3]. These parameters set the bound on the performance and determine both the achievable rate and magnitude of the random-coding error exponent with practical modulation/coding schemes. This letter evaluates the cutoff rate for TH-PPM UWB multiple-access channels.

\section{RANDOM CODING ERROR EXPONENT}

The ensemble average probability of block decoding error using a maximum likelihood decoding is bounded by

$$
P_{e} \leq \exp \left(-N_{b l k}\left[E_{0}(P(x), \rho)-\rho R\right]\right),
$$

where $N_{b l k}$ is the code block length, and $R$ is the information rate per channel symbol forming the ensemble of $\left(N_{b l k}, R\right)$ block codes in which each alphabet is selected with probability $P(x)$. The argument $E(R, \rho)=\left[E_{0}(P(x), \rho)-\rho R\right]$ is known as the channel random coding error exponent [2] where $\rho$ and $P(x)$ are chosen such that maximum exponent value is obtained, because (1) indicates that for some given codes with the same complexity (measured through $N_{b l k}$ ) and same rate

Manuscript received November 25, 2004. The associate editor coordinating the review of this letter and approving it for publication was Prof. Gianluca Mazzini. This research was supported by a grant from NSERC (Natural Sciences and Engineering Research Council), Canada.

The authors are with the Broadband Communications and Wireless Systems (BCWS) Centre, Department of Systems and Computer Engineering, Carleton University, Ottawa, Canada (e-mail: \{adinoyi, halim\}@ sce.carleton.ca).

Digital Object Identifier 10.1109/LCOMM.2005.07007.
$(R)$, the channel having the largest $E(R, \rho)$ value will result in the lowest error probability.

In this paper $\rho=1$ is of interest and is referred to as the cutoff rate, $R_{0}\left(=E_{0}(P(x), 1)\right)$, which can be expressed as [3]

$$
R_{0}=\max _{P_{j}}\left\{-\ln \left[\sum_{j} \sum_{k} P_{j} P_{k} \int_{\mathbf{r}} \sqrt{p(\mathbf{r} \mid j) p(\mathbf{r} \mid k)} d \mathbf{r}\right]\right\},
$$

where $P_{j}$ is the a priori input probability, $p(\mathbf{r} \mid j)$ is probability density function of the output vector given that $j$-th signal was transmitted. If $\log _{2}$ is used instead of $\ln$ in (2), $R_{0}$ is in bits/transmitted waveform. Cutoff rate has been considered the practical capacity beyond which communication would be very expensive. Even though the recent experience with the near-capacity performance of turbo codes (TC) appears to threaten this belief, a substantial amount of price in terms of complexity and delay is still paid through long interleaver and iterative decoding of TC. In sequential decoding applications, cutoff rate remains a valuable parameter which provides insight complementary to that acquired by the study of capacity [4].

Let us consider a time hopping $K$-user UWB system employing M-ary PPM. A typical $k^{\text {th }}$ user's received signal with perfect power control takes the form [1], [5]

$$
s^{(k)}(t)=\sqrt{\frac{E_{s}}{N_{s}}} \sum_{j=0}^{N_{s}-1} p_{r x}\left(t-j T_{f}-c_{j}^{(k)} T_{c}-d_{j}^{(k)}\right),
$$

then the total multiple-access received signal can be represented as

$$
r(t)=s^{(1)}\left(t-\tau^{(1)}\right)+\sum_{k=2}^{K} s^{(k)}\left(t-\tau^{(k)}\right)+n(t),
$$

where user 1 is the user of interest. In (3) and (4), $p_{r x}(t)$ is the basic pulse with a duration of $T_{p}, T_{f}$ is the frame time, $T_{s}=N_{s} T_{f}$ is the symbol duration, $c_{j}^{(k)}$ is the hopping code, $d_{j}^{(k)} \epsilon\left\{\delta_{1}, \cdots, \delta_{M}\right\}$ represents the PPM time shift corresponding to the modulating data sequence of user $k$ at hop $j, \tau^{(k)}$ represents time asynchronism, $E_{s}$ is pulse (symbol) energy, and $n(t)$ is the additive white Gaussian noise (AWGN) with two-sided power spectral density $N_{0} / 2$.

We assume that the receiver is in perfect synchronism with the user of interest so that the correlation receiver can be implemented. With this assumption, the receiver for the Mary PPM scheme is composed of $M$ filters matched to the template functions $\psi_{i}^{(1)}$, defined as

$$
\psi_{i}^{(1)}(t)=p_{r x}\left(t-\delta_{i}-\tau^{(1)}\right), \quad i=1,2, \cdots, M .
$$




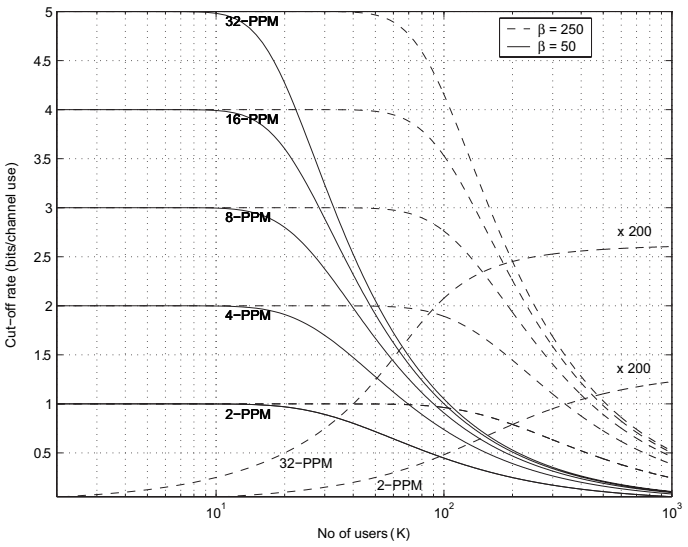

Fig. 1. Cutoff rates of M-ary PPM multiuser UWB for two different spreading factors at $\mathrm{SNR}=20 \mathrm{~dB}$ in the absence of time hopping $\left(N_{s}=1\right)$.

Each matched filter computes as output the following decision statistics

$r_{i}=\sqrt{\frac{N_{s}}{E_{s}}} \sum_{j=0}^{N_{s}-1} \int_{j T_{f}}^{(j+1) T_{f}} r(t) \psi_{i}^{(1)}\left(t-j T_{f}-c_{j}^{(1)} T_{c}-\tau^{(1)}\right) d t$

We can write $r_{i}=D_{i}+I_{i}+N_{i}$ where $D_{i}$ contains the signal to be detected, $I_{i}$ is the multiple-access interference (MAI) and $N_{i}$ is the noise. ${ }^{1}$ The variance of $N_{i}, \sigma_{N_{i}}^{2}$, is obtained as

$$
\sigma_{N_{i}}^{2}=\frac{N_{s}}{E_{s}} \sum_{j=0}^{N_{s}-1} \sum_{k=0}^{N_{s}-1} \delta_{k j} \sigma_{n}^{2} \Gamma(0)=N_{s}^{2} \Gamma(0) \sigma_{n}^{2} / E_{s},
$$

where $\sigma_{n}^{2}=N_{0} / 2$ and $\Gamma(\Delta)$ is the correlation of the basic pulse for a lag $\Delta$. Similarly, $D_{i}=N_{s} \Gamma(0)$, and the MAI part is expressed as

$$
I_{i}=\sum_{k=2}^{K} \sum_{j=0}^{N_{s}-1} \Gamma\left(\Delta_{j}^{(k)}\right)
$$

where $\Delta_{j}^{(k)}=\left(c_{j}^{(1)}-c_{j}^{(k)}\right) T_{c}+\left(d_{j}^{(k)}-d_{j}^{(1)}\right)+\left(\tau^{(k)}-\tau^{(1)}\right)$ is a random time lag between users 1 and $k$ in the $j$-th hop frame. We assume that all the time hopping $\left(c_{j}\right.$ 's) are random, and hence, the monocycle time shift $c_{j}^{(k)} T_{c}$, and the time delays are i.i.d with uniform distribution over a frame interval. Since the UWB pulse duration $T_{p}<<T_{f}$, each interfering pulse contributes to only a single correlation operation (i.e., MAI pulses fall within the same UWB frame). Therefore, $\Delta_{j}^{(k)}$ is uniformly distributed in the interval $\left[-T_{f}, T_{f}\right][6]$. For large values of $K \times N_{s}$, the probability density function (PDF) of $I_{i}$ converges to a Gaussian distribution. Without loss of generality, we consider rectangular monocycle pulse, although, Gaussian and Rayleigh pulses fit easily in the analysis as well. Defining a spreading factor $\beta=T_{f} / T_{p}$, the variance of $I_{i}$ can be expressed, with the help of [6], as

$$
\sigma_{I_{i}}^{2}=\sum_{k=2}^{K} \sum_{j=0}^{N_{s}-1} \operatorname{var}\left[\Gamma\left(\Delta_{j}^{(k)}\right)\right]=(K-1) N_{s}\left(\frac{1}{3 \beta}-\frac{1}{4 \beta^{2}}\right) .
$$

\footnotetext{
${ }^{1}$ The decision statistic for user $1, r_{i}^{(1)}$, is written as $r_{i}$ for notational convenience. The same simplified notation is used in the rest of the letter for other parameters related to user 1 as well, such as $D_{i}, N_{i}$ and $I_{i}$.
}

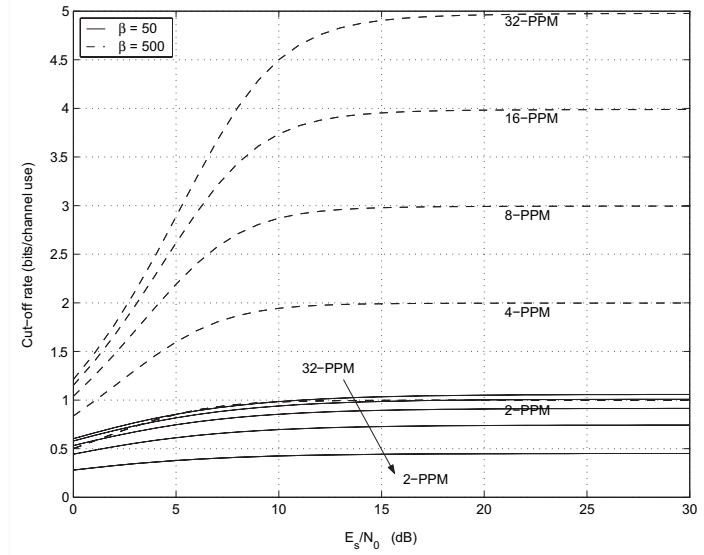

Fig. 2. Cutoff rates of M-ary PPM multiuser UWB for two different spreading factors for $K=100$ in the absence of time hopping $\left(N_{s}=1\right)$.

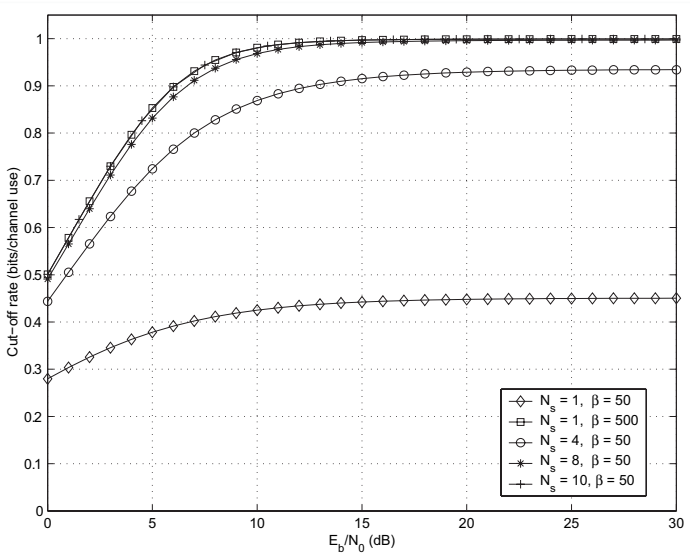

Fig. 3. Cutoff rates of TH 2-PPM multiuser UWB for different spreading and hopping factors for $K=100$.

It can be shown, by exploiting the orthogonality in the PPM M-ary signal set, that the distribution of the received signal $\mathbf{r}$ is given as

$$
\begin{aligned}
p\left(\mathbf{r} \mid \mathbf{x}_{j}\right) & =\left(\frac{1}{2 \pi \sigma_{\text {tot }}^{2}}\right)^{M / 2} \exp \left[-\frac{\left(r_{j}-m_{p}\right)^{2}}{2 \sigma_{\text {tot }}^{2}}\right] \\
& \times \prod_{\substack{k=0 \\
k \neq j}}^{M-1} \exp \left[-\frac{\left(r_{k}-m_{a}\right)^{2}}{2 \sigma_{\text {tot }}^{2}}\right] \\
& =p(r \mid j) \prod_{\substack{k=0 \\
k \neq j}}^{M-1} p(r \mid k)
\end{aligned}
$$

where, $\mathbf{x}_{j}=\left[x_{0}, \cdots, x_{j}, \cdots, x_{M-1}\right]$, is the transmitted signal and $\mathbf{r}=\left[r_{0}, \cdots, r_{j}, \cdots, r_{M-1}\right]$. In the above, $p(r \mid j)$ and $p(r \mid k)$ indicate the PDFs of the matched filter output when the desired signal is present and absent, respectively. The second PDF represents noise-only output. The parameter $\sigma_{\text {tot }}^{2}$ denotes the total noise variance, $m_{p}$ and $m_{a}$ represent slot signal strengths when signal is present and absent, respectively. Using (10) with (2) and $P_{j}=1 / M, 0 \leq j \leq M-1$, the uniform input distribution that maximizes $R_{0}$ [7], the cut-off 
TABLE I

CODE LENGTHS FOR PPM SIGNALING AT RATE 2.0 BITS/SYMBOL

\begin{tabular}{||c|c|c|c|c|c|c||}
\hline \hline & \multicolumn{2}{|c|}{8 -PPM } & \multicolumn{2}{c|}{$16-\mathrm{PPM}$} & \multicolumn{2}{c|}{$32-\mathrm{PPM}$} \\
\hline Number of users & $P_{e} \leq 10^{-3}$ & $P_{e} \leq 10^{-6}$ & $P_{e} \leq 10^{-3}$ & $P_{e} \leq 10^{-6}$ & $P_{e} \leq 10^{-3}$ & $P_{e} \leq 10^{-6}$ \\
\hline 5 & 11 & 21 & 6 & 11 & 4 & 7 \\
\hline 15 & 14 & 28 & 7 & 14 & 5 & 10 \\
\hline 20 & 20 & 39 & 10 & 19 & 7 & 14 \\
\hline 30 & 107 & 214 & 21 & 42 & 14 & 28 \\
\hline \hline
\end{tabular}

rate $R_{0}$ can be expressed as

$$
\begin{aligned}
& R_{0}=\max _{P_{j}}\left\{\begin{array}{l}
-\log _{2}\left[\sum_{l=0}^{M-1} P_{l}^{2}\left(\int_{-\infty}^{\infty} \sqrt{p(r \mid l) p(r \mid l)} d r\right)^{2}\right. \\
\left.+\sum_{k=0}^{M-1} \sum_{\substack{j=0 \\
j \neq k}}^{M-1} P_{j} P_{k}\left(\int_{-\infty}^{\infty} \sqrt{p(r \mid j) p(r \mid k)} d r\right)^{2}\right] \\
=\log _{2}(M)-\log _{2}\left[1+(M-1) \exp \left(-\frac{\left[m_{p}-m_{a}\right]^{2}}{4 \sigma_{\text {tot }}^{2}}\right)\right]
\end{array}\right.
\end{aligned}
$$

where $m_{p}=E\left[r_{i} \mid\right.$ given that the desired signal is present $]=$ $N_{s} \Gamma[0], m_{a}=E\left[r_{i} \mid\right.$ given that the desired signal is absent $]=$ $E\left[I_{i}+N_{i}\right]=0$, and $\sigma_{\text {tot }}^{2}=\sigma_{N_{i}}^{2}+\sigma_{I_{i}}^{2} . E[\cdot]$ denotes the mean operator.

Finally, we observe that when the network becomes heavily loaded, the cutoff rate asymptotically approaches zero, but the aggregate rate $R_{0, a g g}\left(=K R_{0}\right)$ converges to a nonzero constant; this is confirmed by the asymptotic aggregate cutoff rate expression derived as

$$
\begin{aligned}
R_{o, a g g} & =\lim _{K \longrightarrow \infty} K \log _{2}\left[\frac{M}{1+(M-1) \exp \left(-\frac{\left[m_{p}-m_{a}\right]^{2}}{4 \sigma_{\text {tot }}^{2}}\right)}\right] \\
& \approx \frac{3 \beta(M-1)\left(m_{p}-m_{a}\right)^{2}}{2 N_{s} M \log _{e}(2)} .
\end{aligned}
$$

\section{NUMERICAL RESULTS}

Fig. 1 shows the cutoff rate of the UWB system versus number of users and also shown is the asymptotic behaviour of the aggregate cutoff rate for 2- and 32-PPM. We observe that a certain maximum number of users can be accommodated in order to achieve the maximum $R_{0}\left(=\log _{2}(M)\right)$. It is further observed that using a higher spreading factor delays the fast drop in the cutoff rate with respect to the number of users.

Fig. 2 shows the cutoff rate as a function of SNR for a fixed number of users of $K=100$. We observe that a heavily loaded channel results in an extremely low $R_{0}$ when the spreading factor is relatively low $(\beta=50)$. For example, 32-PPM could only operate at $1 \mathrm{bit} / \mathrm{symbol}$ even at large SNR when $\beta=50$. Increasing the spreading factor yields a better performance (higher $R_{0}$ ), but this is not always desirable because of the potential synchronization problems. The impact of time hopping $\left(N_{s}=4, N_{s}=8\right.$, and $\left.N_{s}=10\right)$ on $R_{0}$ is shown in Fig. 3 for 2-PPM. It is observed that the capacity of TH system $\left(N_{s}=4\right)$ is approximately twice that of a nonhopping system $\left(N_{s}=1\right)$ for $\beta=50$.

\section{A. Coding Complexity Measure}

Consider that a waveform with a rate of 2 bits/symbol is desired. A natural choice would have been 4-PPM, but Fig. 2 indicates that 4-PPM and 8-PPM require about $12 \mathrm{~dB}$ and 4 $\mathrm{dB}$, respectively, for $\beta=500$. Therefore, 8-PPM has a power saving advantage if a suitable coding scheme with certain complexity, in Shannon sense, can be found for a given error rate.

Table I shows the computed block lengths (complexity) required to satisfy a specific probability of error for schemes operating at 2 bits/symbol and $10 \mathrm{~dB}$ SNR per user. The results for $\beta=50$ and $N_{s}=1$ are given. We found that significant increase in code lengths is required in the presence of high amounts of MAI for 8-PPM as compared to 16-PPM and 32PPM. Also, we observed that only a two-fold increase in the block length results in an error rate reduction from $10^{-3}$ to $10^{-6}$ for all the M-ary schemes. A further investigation of the actual coding scheme for the system described in this letter can be undertaken. The work on capacity limit achievable by Reed-Solomon (R-S) M-ary PPM in AWGN presented in [7] can complement this effort.

\section{CONCLUSION}

This work evaluates the practical capacity, known as the cut-off rate, for TH-PPM adopted for UWB communication over multiple-access channels, without a need for numerical integration or Monte Carlo simulation. We have shown how the cutoff rate can be used in M-ary PPM UWB multipleaccess communication systems for determining the system trade-offs. Moreover, cutoff rate evaluated in this letter can be a fast way of gaining insights into the multiuser capacity of TH-PPM UWB systems.

\section{REFERENCES}

[1] M. Z. Win and R. A. Scholtz, "Ultra-wide bandwidth time-hopping spread-spectrum impulse radio for wireless multiple-access communications," IEEE Trans. Commun., vol. 48, pp. 679-691, Apr. 2002.

[2] W. K. Ahmed and P. J. McLane, "Random coding error exponents for flat fading channels with realistic channel estimation" IEEE J. Select. Areas Commun., vol. 18, pp. 369-379, Mar. 2000.

[3] J. Davidson and I. Kalet, "Bhattacharyya bound, cutoff rate, and constellation design for the companding channel," IEEE Trans. Commun., vol. 45, pp. 770-787, July 1997.

[4] E. Biglieri, J. Proakis, and S. Shamai, "Fading channels: Informationtheoretic and communications aspects," IEEE Trans. Inform. Theory, vol. 44, pp. 2619-2692, Oct. 1998.

[5] B. Hu and N. Beaulieu, "Exact bit error rate analysis of TH-PPM UWB systems in the presence of multiple-access interference," IEEE Commun. Lett., vol. 7, pp. 572-574, Dec. 2003.

[6] M. Abdel-Hafez, F. Alagoz, and M. Hamalainen "Performance of M-ary PPM UWB radio in fading channels," J. Commun. Networks, vol. 5, pp. 365-373, Dec. 2003.

[7] S. Dolinar, D. Divsalar, J. Hamkins, and F. Pollara, "Capacity of pulseposition modulation (PPM) on Gaussian and Webb channels," JPL/NASA TMO progress report 42-142 (JPL), Aug. 2000 (available online at http://tmo.jpl.nasa.gov/tmo/progress_report/tmo.html). 